\title{
Aumento da procura de tratamento por usuários de crack em dois ambulatórios na cidade de São Paulo, nos anos de 1990 a 1993
}

\author{
C.P. Ferri, R.R. Laranj EIRA, D.X. DA Silveira, J . Dunn, M.L.O.S. Formigoni
}

Departamentos de Psiquiatria e de Psicobiologia, Universidade Federal de São Paulo - Escola Paulista de Medicina, São Paulo, SP.

\begin{abstract}
RESUMO - Nos últimos anos tem sido observado aumento do uso de crack (uma forma de cocaína fumada) em pesquisas epidemiológicas e em apreensões policiais. Até o presente, não havia dados brasileiros relacionando a procura de tratamento para a dependência de cocaína com as vias habituais de administração
\end{abstract}

Овј etıvo. Analisar as modificações das vias de administração da cocaína em uma população de 245 pacientes atendidos em dois serviços ambulatoriais especializados (PROAD e UDED), na cidade de São Paulo, entre os anos de 1990 e 1993.

Métodos. Dados de entrevistas padronizadas realizadas na admissão dos pacientes aos serviços

\section{INTRODUÇÃO}

Nos últimos anos, uma nova forma da cocaína tem-se tornado disponível em nosso meio. Esse produto, denominado crack, é uma forma potente de cocaína que resulta em rápido e notável efeito estimulante quando fumado. A euforia ocorre dez segundos após a inalação, com o pico de concentração plasmática da cocaína atingido entre 5 e 10 mi nutos após a inalação $0^{1,2}$. Concentrações semel hantes só são atingidas após uma hora da administração intranasal de uma dose equival ente ${ }^{3}$. O crack é resultado da adição de bicarbonato de sódio e adulterantes ao cloridrato de cocaína ("pó"). Após o aquecimento dessa mistura, obtém-se um resíduo seco que é vendido na forma de pequenas "pedras" que podem ser fumadas em cachimbos, cigarros e outros objetos improvisados. O nome crack provém do barulho que é produzido pela quebra dessas "pedras". Quando fumado, o crack produz pequenas partículas que são absorvidas rapidamente pelos pulmões, conduzindo imediatamente ao aparecimento dos efeitos. A velocidade desse processo parece ser um dos fatores responsáveis pelo seu alto poder de adição $0^{4,5}$.

Um dos aspectos muito importantes no uso do crack éa dimensão dos problemas físi cos associados. No trato respiratório, têm sido observados vários foram estudados e determinada a prevalência de uso das diferentes vias de administração de cocaína.

Resultados. A percentagem de pacientes que relataram uso de cocaína fumada (crack) aumentou de $17 \%$, em 1990, para 64\%, em $1993(p<0,01)$. O uso de cocaína aspirada não variou durante esse período, permanecendo a via mais freqüentemente relatada (80\%), enquanto a via endovenosa variou de $40 \%$, em 1990, para 18\%, em 1992, e para 28\%, em 1993.

Conclusões. As implicações do aumento de usuários de crack que procuram tratamento são discutidas em função do planejamento de tratamento e de programas de prevenção, com ênfase no risco de transmissão do vírus HIV.

problemas como: tosse, expectoração enegrecida, dor peitoral, redução da função pulmonar, com capacidade de expiração comprometida e, em casos mais graves, pneumotórax espontâneo e enfisema no mediastino ${ }^{6}$. No aparel ho cardi ovascular, o aumento da freqüência cardíaca e da PA e o notável efeito vasoconstritor podem levar a uma parada cardíaca. Outros efeitos associados ao uso de crack são necrose muscular, problemas neurológicos como convulsões e hemorragias cerebrais, e problemas psiquiátricos como paranóia, depressão severa e ataques de pânico. Alguns estudos detectaram importantes alterações neurológicas nos filhos de usuárias de crack, como retardo no crescimento intra-uterino, menor perímetro cefálico, tremores, irritabilidade, rigidez muscular e convulsões transitórias ${ }^{7}$.

Nos Estados Unidos, o uso do crack tornou-se popular em meados dos anos 80 . Seu desenvol vimento deu-se de forma cíclica durante aquela década, tendo atingido o pico de consumo por volta de $1990^{\circ}$. Nesse período, inúmeros trabalhos foram publicados na literatura internacional a respeito do crescimento dessa via de administração da cocaína, dos seus efeitos no organismo, assim como das características particulares dos seus usuários, sua relação com criminalidade ${ }^{9}$, comportamento sexual e influência no risco de transmissão da AIDS ${ }^{10}$. 
Em revisão publicada recentemente ${ }^{11}$, observase que, no Brasil, a cocaína tem despertado interesse cada vez maior da mídia e dos pesquisadores, nos últimos anos. Em relação ao crack, apesar de indícios do seu consumo crescente, poucos trabalhos têm sido desenvolvidos no sentido de entender esse uso, potencialmente, tão comprometedor. No mais recente levantamento real izado pelo Centro Brasileiro de I nformações sobre Drogas (CEBRID), setor do Departamento de Psicobiologia da EPM, entre estudantes ${ }^{12}$ e meninos de rua ${ }^{13}$, parece nítido o aumento do uso de cocaína, em todo o Brasil, com destaque para São Paulo e Rio de J aneiro. O crescimento do uso de crack é muito mais evidente em São Paulo do que nas outras cidades, havendo diferença importante na prevalência do uso dessa droga (cocaína) e das suas formas de administração nas diversas regiões do país. $\mathrm{Na}$ Região $\mathrm{N}$ ordeste, por exemplo, o uso de cocaína é muito pequeno entre os estudantes.

Tendo em vista a carência de informações sobre o uso de crack no Brasil, este trabalho visou estudar a prevalência de uso de cocaína e suas vias de administração, na população atendida em dois serviços ambulatoriais para tratamento de dependentes de drogas de São Paulo, nos anos de 1990 a 1993.

\section{MÉTODOS}

Foram anal isados os registros dos pacientes atendidos em dois serviços ambulatoriais públicos da cidade de São Paulo, no período de 1990 a 1993. Os dois ambulatórios estudados (PROAD e UDED) são setores especializados no tratamento de abuso e dependência de drogas. O PROAD (Programa de Orientação e Atendimento a Dependentes de Drogas) é um setor do Departamento de Psiquiatria da Escola Paulista de Medicina (EPM), ea UDED (Unidade de Dependência de Drogas), um setor do Departamento de Psicobiologia da EPM. Ambos oferecem tratamento gratuito.

As duas clínicas usam uma entrevista inicial padronizada, que, embora em sua forma e aplicação sejam diferentes, possuem algumas variáveis em comum, permitindo a análise dos dados agrupados. Foram estudados: idade e estado civil, tipo de drogas e vias utilizadas para o consumo. Para a análise dos dados foi utilizado o teste do $\chi^{2}$ de tendência.

Foram estudados 245 pacientes com diagnóstico de abuso e dependência de cocaína no momento da entrevista inicial, de acordo com os critérios do DSMIII-R. Eles correspondiam a cerca de $50 \%$ da população total de dependentes atendida no PROAD e na UDED. A média de idade da amostra

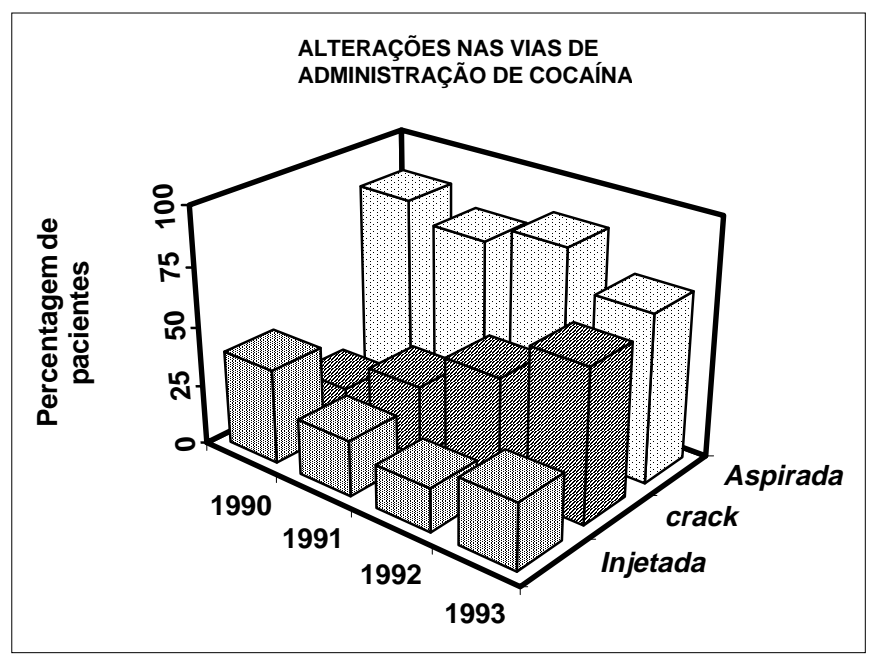

Fig. - Vias de admi nistração de cocaína em dependentes queprocuraram tratamento nos anos de 1990 a 1993. ( $\chi^{2}$ de tendênci $a=25,6 ; p<0,01$.)

foi de 24,8 anos (variando de 13 a 45 anos). Oitenta e nove por cento eram homens, sendo $68 \%$ sol teiros, $24 \%$ casados e $8 \%$ separados ou divorciados.

\section{RESULTADOS}

A fig. mostra a percentagem de usuários de cocaína que relatam uso de quaisquer uma das três vias de administração (fumada, aspirada e injetada). Alguns usuários utilizavam mais de uma via de administração, o que leva a uma percentagem total maior do que $100 \%$. Podemos observar um aumento significativo da cocaína fumada $(p<0,01)$ no período de 1990 a 1993, um nível relativamente alto e estável da cocaína aspirada ao longo dos quatro anos e uma flutuação na percentagem de usuários da via endovenosa de 40\%, em 1990, para $18 \%$, em 1992, e 28\%, em 1993.

A duração do uso de cocaína era registrada de forma diferente pelos dois serviços. Em uma das clínicas (PROAD) registrava-se o tempo decorrido entre o primeiro contato com a droga e a procura daquel e tratamento, que foi de 6,6 anos, em média, variando de 1 a 21 anos. Na outra clínica (UDED) era registrado o tempo de uso regular, que foi de 7,9 anos, em média, variando entre 1 e 25 anos.

\section{DISCUSSÃO}

O estudo do padrão de consumo de drogas em dependentes é de fundamental importância para a compreensão da natureza dos distúrbi os decorrentes desse uso, bem como para a determinação das políticas de saúde (prevenção e tratamento) dirigidas a esse problema. 
Alguns estudos de literatura alertam para as limitações nos estudos de populações específicas, como é o caso dos usuários de drogas que se encontram em tratamento. Estes indivíduos parecem apresentar uma diferença significativa quanto ao padrão de uso e outras características, quando comparados a populações de dependentes que não estão em tratamento ${ }^{14}$. No entanto, esses dados são úteis como indicadores indiretos, sendo, freqüentemente, utilizados para avaliar a prevalência de usuários com uso abusivo ${ }^{15}$. Em relação ao crack especificamente, o padrão de uso e outras características parecem diferir, quando comparados não apenas com populações que não estão em tratamento, mas, também, com populações que fazem uso abusivo de outras drogas ${ }^{16}$.

Apesar de haver várias publicações nacionais recentes sobre cocaína ${ }^{11}$, a maioria dos estudos se preocupa com questões epidemi ol ógicas em populações específicas, como meninos de rua, estudantes ou pacientes em tratamento. Pouco se sabe a respeito do padrão de uso e das características específicas dos usuários.

O aumento da procura de tratamento por usuários de crack poderia estar relacionado com a aparente tendência de diminuição da procura de tratamento por usuários da via injetável. No entanto, seriam necessários estudos específicos para avaliar essa questão. Por outrolado, estudos realizados nos EUA detectaram indícios de uma forte associação entre uso do crack e transações de sexo por drogas ou dinheiro ${ }^{17}$. Desta forma, embora, considerando a via de administração, o usuário de crack pudesse ter menor risco de contaminação pelo HIV, esse risco poderia estar aumentado pelo comportamento sexual de risco. Considerando que no Brasil, até maio de 1994, haviam sido notificados 11.485 casos de homens infectados em razão do uso de drogas e 13.905 infectados por relações homossexuais, torna-se urgente avaliar o potencial disseminador do vírus HIV pelo comportamento sexual de risco dos usuários de drogas ${ }^{18}$.

Este trabalho detectou um aumento da procura de tratamento por usuários de crack nos últimos quatro anos, em dois serviços públicos. Esse aumento ressal ta a necessidade de um conhecimento mais amplo das características desse tipo de usuário que permita uma abordagem terapêutica mais eficien$\mathrm{te}^{19}$. Alguns dados de literatura indicam que a aderência desses usuários a programas de tratamento é ainda menor que a de abusadores de outras drogas ou de cocaína por outra via de administração ${ }^{20}$.

Estudos mais detalhados sobre o comportamento dessa população são necessários, consi derando que cada subgrupo de usuários de cocaína apresenta especificidades que podem ser fundamentais no planejamento das políticas de tratamento e prevenção.

Trabalho realizado com apoio financeiro da AFIP (Associação Fundo de Incentivo à Psicofarmacologia).

\section{SUMMARY}

\section{Increase in crack users attending treatment services in São Paulo: 1990-1993}

An increase in crack use has been detected in epidemiological research and policedata. Currently, in Brazil, no data are available linking the route of administration and attendance to treatment for cocaine dependence

Objective - The purpose of this paper was to analyze the changes in cocaine routes of administration in a cocaine dependent population treated in two outpatient public services (PROAD and UDED).

Method - Standardized interview data, coll ected at admission to treatment were compared from 1990 to 1993. The prevalence rates of smoked ("crack"), injected and snorted cocaine were compared.

Results - The percentage of patients who reported "crack" cocaine use increased from 17\% in 1990 to $64 \%$ in $1993(p<0.01)$ The prevalence of snorted cocaine remained stable in the period of time analyzed, being the most frequent route reported. The intravenous route tended to decrease from $40 \%$ in 1990 to $28 \%$ in 1993.

Concl usion - The implications of the increase of "crack" cocaine users who sought treatment are discussed. These data are important in planning prevention and treatment strategies, mainly in AIDS prevention. [ Rev Ass Med Brasil 1997; 43 (1): 25-8.]

\section{REFERÊNCIAS BIBLIOGRÁFICAS}

1. J ones RT. The pharmacology of cocaine. In Grabowski J (ed): Cocaine: pharmacology, effects and treatment abuse. Rockville, Maryland; NIDA Research Monograph 50, 1984.

2. Kleber HD, Gawin FH. The spectrum of cocaine abuse and its treatment. J Clin Psychiatry 1984; 45: 18-23.

3. Van Dyke C, Byck R. Cocaine. Scient Amer 1982; 246: 128-41.

4. Cox TC, J acobs MR, LeBlanc AE, Marshman J A. Drugs and drug abuse - a reference text. 2nd ed, Toronto, Addiction Research Foundation, 1987; 240-9.

5. Inciardi J A. Beyond cocaine: basuco, crack and other coca products. Contemp. Drug Probl 1987; 14: 461-92.

6. Khalsa ME, Tashkin DP, Perrochet B. Smoked cocaine: patterns of use and pulmonary consequences. Psychoactive Drugs 1992; 24: 265-72.

7. Smart RG. Crack cocaine use: a review of prevalence and adverse effects. Am J Drug Alcohol Abuse 1991; 17: 13-26. 
8. Hamid A. The development cycle of a drug epidemic: the cocaine smoking epidemic of 1981-1991. Psychoactive Drugs 1992; 24: 337-48.

9. Inciardi J A, Pottieger AE. Kids, Crack and Crime. J Drug Issues 1991; 21: 257-70.

10. Bowser BP. Crack and AIDS: an ethnograficimpression. J Natl Med Assoc 1989; 81: 538-40.

11. Carlini EA, Nappo SA, Galduróz J C. A cocaína no Brasil ao longo dos últimos anos. Rev ABP-APAL 1993; 15: 121-7.

12. Galduróz J CF, D’Almeida V, Carvalho V, Carlini EA. III Levantamento sobre o uso de drogas entre estudantes de 10 e 2 graus em 10 capitais brasileiras em 1993. São Paulo, CEBRID-Departamento de Psicobiologia-EPM, 1994.

14. Pottieger AE, Tressel PA, Inciardi J A, RosalesTA. Cocaine use patterns and overdose. Psychoactive Drugs 1992; 24 399-410.
15. Kandel DB. The social demography of drug use. Milbank Q 1991; 69: 365-414.

16. Lewis C, J ohnson BD, Golub A, Dunlap E. Studying crack abusers: strategies for recruting the right tail of an ill-defined population. Psychoactive Drugs 1992; 24: 323-36.

17. Wolfe H, Vranizan KM, Gorter RG et al. Crack use and human immunodeficiency virus infection among San F rancisco intravenous drug users. Sex Transmit Dis 1992; 19: 111-4.

18. Ministério da Saúde. AIDS Boletim E pidemiológico, 1994 Ano VII, no 5 .

19. Wallace BC. Treating crack cocaine dependence: the critical role of relapse prevention (review). Psychoactive Drugs 1992; 24: 213-22.

20. Ferri CP, De Micheli D, Formigoni MLOS. O que diferencia o usuário de cocaína fumada ("crack") dos usuários de cocaína por outras vias. (submetido a publicação). 